\title{
EMOZIONE E REALTÀ NELL'IMMAGINE DI PLATONE
}

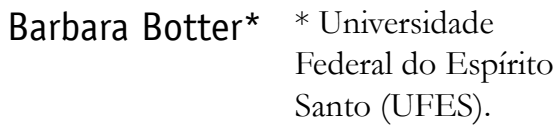

ESTRATTO: In Platone, l'immagine è rappresentazione pervasa di emozione. Non esiste immagine, neppure una semplice copia, che non contenga un'emozione e non esiste una pittura, neppure la più astratta, che non rappresenti un'idea. Il fine del presente articolo è di proporre una lettura e interpretazione del concetto di immagine in Platone, che mantenga vivi i due aspetti costitutivi dell'immagine, la carica emotiva e il riferimento al contenuto reale di cui l'immagine è rappresentazione. Il testo è diviso in tre sezioni: nella prima e nella seconda sezione indago lo statuto ontologico dell'immagine e la sua relazione con l'archetipo; nella terza parte propongo una interpretazione della funzione dell'immagine in Platone e in alcuni passi della letteratura greca antica.

PAROLE CHIAVE: immagine; modello; identità; differenza; mimesis.

\section{EMOTION AND REALITY IN PLATO'S IMAGE}

\begin{abstract}
In Plato, the image is representation peppered with emotion. There is no image, not even a simple copy, that does not contain a minimum of emotion and there is no painting, not even the most abstract, that does not represent a reality or an idea. The aim of this article is to suggest an interpretation of the concept of image in Plato, which keeps the two fundamental aspects of the image alive: its emotional content and the reference to a real content of which the image is image. The text is divided into three parts: the first and the second part investigate the ontological statute of the image and its relationship with the archetype. The third section offers an interpretation of the image's function of image in Platonic and Greek Literature.
\end{abstract}

KEYWORDS: image; original; identity; difference; mimesis.

\section{INTRODUZIONE}

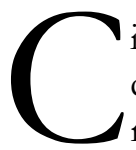

ircola tra gli esegeti di Platone un aneddoto sul grande esperto di studi classici Ulrich von Wilamowitz-Moellendorf. Si racconta che quando lo studioso espresse ai colleghi più anziani dell'Accademia di Berlino il desiderio di scrivere un libro su 
un'interpretazione complessiva del pensiero di Platone, essi rimasero scandalizzati: -forse un'opera sull'uso del caso dativo nei dialoghi della gioventù- azzardarono alcuni, -ma un testo sull'intera filosofia di Platone è, anzitutto, impertinente, in secondo luogo, impossibile (GERSON, 1990, p. IX).

Uno studio che pretendesse di analizzare il concetto di immagine ${ }^{1}$ (eidolon) nell'opera di Platone, si pone esattamente al lato di quelle proposte che farebbero inorridire i membri dell'Accademia di Berlino. Senza esagerare, possiamo dire che, in tutti i dialoghi di Platone sono presenti, più o meno velate, allusioni all'arte mimetica e alla mimesis. ${ }^{2}$

Platone stesso è un creatore di immagini (mimetes) $[$ di dialoghi platonici sono creazioni letterarie di carattere eminentemente artistico, in cui l'aspetto dottrinale e quello drammatico sono indissolubilmente legati. Il filosofo ateniese riproduce nei suoi dialoghi situazioni fittizie che rimandano a strutture drammaturgiche tratte dalla commedia, ${ }^{3}$ e "l'interpretazione del testo deve tenere conto dell'azione del dialogo, che va letto come un dramma all'interno del quale l'azione e il tema sono strettamente legati e si illuminano a vicenda" (PERINE 2011, p. 207).

La mimesis appare ancora a livello ontologico. Nella Repubblica e nel Timeo, la dimensione empirica, tanto umana quanto cosmica, è considerata una scena, dietro alla quale si cela la dimensione ideale, ossia la struttura intelligibile degli enti (PALUMBO, 2008).

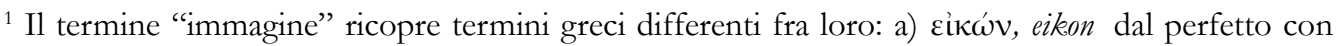
senso di presente del verbo eoika, generalmente tradotto con "sono simile, somiglio, sembro, ho

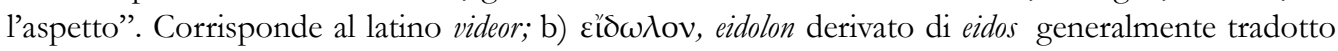

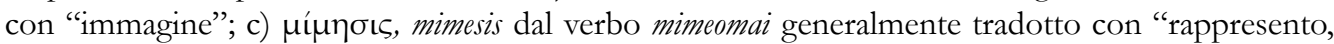
riproduco imitando". Il sostantivo da esso derivato, viene generalmente tradotto con "imitazione"; d) $\phi \alpha ́ v \tau \alpha \sigma \mu \alpha$, phantasma dal verbo phantazo generalmente tradotto con "parvenza, apparenza, immagine".

${ }^{2}$ Democrito fu il primo ad usare il termine mimesis nel frammento $154 \mathrm{DK}$ : "e noi siamo proprio, per le attività più importanti, allievi degli animali, e precisamente (...) degli uccelli dal canto melodioso e del cigno e dell'usignolo nel canto kata mimesin". Cf. PALUMBO, 2011, p. 168, n. 39. Sul significato del termine mimesis nella cultura antica si veda POLLITT, 1974; SÖRBOM, 1966 e 2002, p. 19-27; HALLIWELL, 2002. Per uno studio approfontido della mimesis in Platone ed Aristotele rimando all'eccellente opera di Lidia PALUMBO, 2008 e agli altri numerosi saggi dell'autrice sull'argomento. Sono profondamente debitrice agli studi di Lidia Palumbo sulla mimesis per molte delle informazioni che seguono in questo testo e per la ricca e preziosa bibliografia utilizzata e indicata nelle opere della studiosa, che è stata molto preziosa per il mio studio. La traslitterazione dei termini greci rispetta le Novas Normas de Transliteração pubblicate nella rivista Archai, n. 12, p. 193-194 e disponibili nel link: <http://periodicos.unb.br/index.php/archai/article/view/10149/7457>.

${ }^{3}$ HAMM osserva "Já o tradutor e organizador da primeira edição das obras de Platão, Otto APELT, publicou em 1912 um trabalho sobre o cômico em Platão e WILAMOWITZ-MOELLENDORF, grande conhecedor de Platão e contemporâneo de Apelt, qualificou o Banquete e a Apologia de puras comédias" (2014, p. 65, n.1). Lo stesso può dirsi di molte altre opere, come il Protagora e il Fedro. Sull'aspetto drammatico di queste opere, si veda CASERTANO, 2004, p. 728-766, LASPIA, 2011, p. 95-111 e SPINA, 2005, p. 198-209.
} 
Infine, sulla base della classificazione che il proprio Platone fornisce dei generi letterari nel libro III della Repubblica, il filosofo designa col termine mimesis un tipo di narrazione, la narrazione dia mimeseos (392d5), ${ }^{4}$ Che si ha quando il narratore parla in prima persona vestendo i panni di un personaggio. Come nota Casertano (2011, p. 10), questo tipo di narrazione può essere attribuito agli scritti dello stesso Platone. ${ }^{5}$

Non ho la pretesa di affrontare in modo compiuto la complessa questione dell'arte mimetica in Platone. Inoltre, su una questione come questa, "La bêtise consiste a vouloir conclure", direbbe Flaubert. Ma c'è conclusione e conclusione. Se è ridicolo pretendere di trattare in modo esaustivo il concetto e i contenuti della mimesis in Platone, non lo è percorrere un certo itinerario, cercando di riportare alla luce i significati reconditi di un tema la cui ricchezza è profonda.

Scopo del presente testo è di proporre un'interpretazione del contenuto della mimesis in Platone che non riduca questo ricco concetto all'atto di imitazione, come viene per lo più interpretato. I significati dell'imitazione e dell'imitare riportati tradizionalmente nel dizionario sono "fare a somiglianza di altri", "prendere ad esempio", "ritrarre", "contraffare". In tali accezioni, tutte legittime, non si evince la potente carica emotiva che abita la semantica della mimesis così come essa è concepita nei dialoghi di Platone. Al contrario, come già riconosciuto da Palumbo (2011, p. 166, n. 32), questa traduzione è inopportuna, da un lato, per il fatto che ignora alcuni tratti essenziali dell'immagine in Platone e, dall'altro, perché include alcune caratteristiche di cui la mimesis platonica è priva.

Per attingere il mio obiettivo, dividerò il mio testo in tre itens. Nella prima sezione condurrò un'indagine sullo statuto ontologico dell'immagine. ${ }^{7}$ Nella seconda parte indagherò la relazione fra l'immagine e il modello di cui l'immagine è immagine. Infine, nell'ultimo item metterò a confronto alcuni passi dei dialoghi platonici con passi tratti della letteratura greca antica dai quali si evince la ricchezza di contenuto del termine greco, nel tentativo di stabilire un'espressione che mantenga la ricchezza del contenuto della mimesis e degli elementi di cui la mimesis è intessuta eikon e eidolon. ${ }^{8}$

\section{1) Lo STATUTO ONTOLOGICO DELL'IMMAGINE}

Platone usa il lessico della mimesis nell'analisi poetica e per indicare dimensioni della realtà molto diverse tra loro: per definire la relazione tra sensibile e intelligibile

\footnotetext{
${ }^{4}$ Sul tema si veda PALUMBO, 2011, p. 165.

${ }^{5}$ Il caso della Repubblica è emblematico. La Repubblica è considerata un'opera "diegetico-mimetica", ossia costituita da una narrazione (diegesis), all'interno della quale sono riprodotti i dialoghi così come furono condotti dai vari personaggi (mimesis). Conclude Casertano: "trata-se de uma verdadeira representação teatral” (2011, p. 10). Si veda anche CASERTANO, 2004, p. 731.

${ }^{6}$ Flaubert, apud FOLSCHEID \& WUNENBURGER, 1997, p. 227.

${ }^{7}$ La mia analisi non ha la pretesa di essere esaustiva; utilizzerò solo quei dialoghi e quegli aspetti che sono funzionali ad una più chiara comprensione della natura dell'immagine.

${ }^{8} \mathrm{~A}$ volte Platone usa anche il termine phantasma senso specifico come accade nel Sofista 235c8-236c7, in cui il termine indica un tipo specifico di immagine.
} 
(ambito ontologico); ${ }^{9}$ per indicare il rapporto che esiste tra un termine del discorso e il suo corrispettivo reale, sia essa la realtà dell'idea o la dimensione empirica (filosofia del linguaggio); ${ }^{10}$ e per delimitare uno speficico genere letterario (Repubblica III, 392d5).

Nel dialogo Cratilo, riflettendo sulla natura dei nomi, Socrate fornisce una definizione di immagine che mette in evidenza un aspetto costitutivo di essa e che Gregory Vlastos chiama "principio di non-identità" (1973, p. 87). Questo principio è essenziale per intendere lo statuto ontologico dell'immagine e la relazione tra l'immagine e il suo archetipo. A Cratilo, che definisce una "buona immagine" quella che riproduce nel modo più fedele il suo modello, Socrate ribatte che l'immagine non è una semplice riproduzione dell'oggetto:

Bada che la correttezza per un'immagine nella sua interezza non sia questa, bensì, al contrario, non debba affatto riprodurre tutto di ciò che imita, se dev'essere una immagine. ${ }^{11}$

Il ritratto di Cratilo non è un secondo Cratilo, ma una nitida immagine di Cratilo. ${ }^{12}$ Continua Socrate:

Non vi sarebbero, forse, due cose, quali, per esempio, Cratilo e l'immagine di Cratilo, se un dio, non solo riproducesse il tuo colore e la tua figura, come i pittori, ma anche tutto l'intero, così com'è, e gli assegnasse la stessa mollezza, lo stesso calore, e vi infondesse movimento, anima e intelletto, proprio quali sono in te, e, in una parola, di tutte le tue caratteristiche ti ponesse accanto la copia? In questo caso vi sarebbero, forse, Cratilo e una immagine di Cratilo, oppure due Cratili? - Mi sembra, Socrate, che vi sarebbero due Cratili. ${ }^{13}$

\footnotetext{
${ }^{9}$ In particolare nella Repubblica e nel Timeo, ma anche nel Fedone e nel Fedro. Nel Fedro, il filosofo afferma che le cose di quaggiù altro non sono che homoiomata, immagini delle idee (250a6; b3).

${ }^{10}$ Si veda PALUMBO, 2011, p. 165. Platone riconosce che il discorso è l'unico strumento dato all'uomo per esprimere la realtà. Tuttavia, il rapporto fra linguaggio e realtà non è immediato. Nel Teeteto ci sono evidenti spie della coscienza di questa problematicità (157b; 179e-183b). Per la problematicità della relazione linguaggio-realtà nel Teeteto, si veda CASERTANO, 2000, p. 367-381. Il problema del rapporto fra linguaggio e realtà è evidente in tutti gli ultimi dialoghi di Platone, nel Parmenide, nel Sofista, nel Politico, nel Filebo e nel Timeo. Su questo punto di veda CASERTANO, 1996.

${ }^{11}$ Cratilo 432b-c:

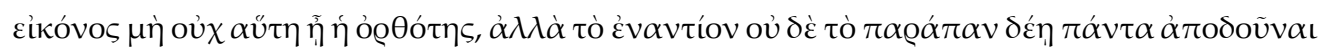

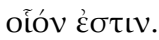

${ }^{12}$ Commenta Rose "An accurate image cannot falsify the proportions of its original, its falsehood must be due to the fact that it is like but not the original" (1986, p. 151).

${ }^{13}$ Cratilo 432c:

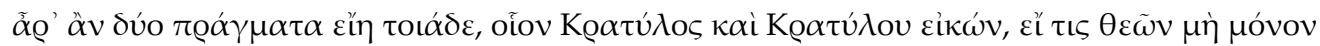

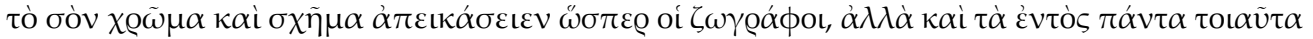

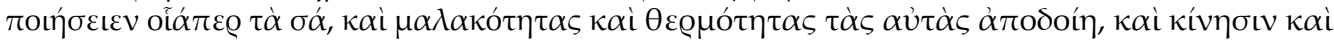

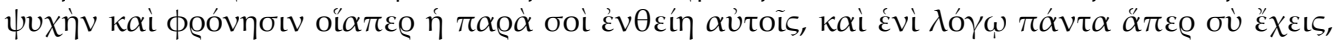

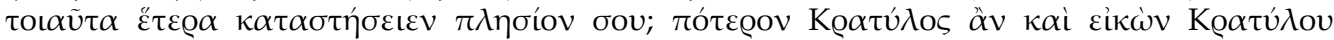

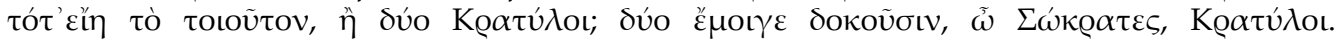
Su questo passo si veda GASTALDI, 2007, p. 93-149.
} 
L'immagine, anche se simile, è irriducibilmente diversa dall'originale: "Credere ciò che è simile (bomoion) non simile, ma identico (auto), è infatti propriamente la condizione di chi sogna", afferma il filosofo nella Repubblica (476c). Nell'immagine, la somiglianza e la differenza con il modello risultano indissolubilmente legate, come si evince dalla definizione di immagine (eidolon) ${ }^{14}$ [hel Sofista (240a). ${ }^{15}$

Che cosa potremmo dire che sia l'immagine (eidolon), se non l'oggetto fatto a somiglianza di quello vero, diverso (heteron) da esso, ma tale e quale (toiouton)? ${ }^{16}$

La definizione è apparentemente chiara e profondamente oscura.

Lo Straneiro di Elea chiede che sia chiarito il significato dell'espressione "diverso, ma tale e quale (toiouton)". ${ }^{17}$

Ma con diverso ma tale e quale, intendi dire un oggetto vero (aletbinon), oppure a cosa riferisci la parola "tale e quale"?

Per niente un oggetto vero, bensì uno somigliante (eoikos)..$^{18}$

L'immagine, (eidolon) non è vera (aletbinon), perché vera è solo la realtà. L'immagine non è realmente (ontos), ossia non è un ente reale. Ma, pur non essendo vera, e perciò non essendo reale, l'immagine è realmente una copia $(e i k o n) .{ }^{19}$ Se vero è solo l'ente reale e non vero

\footnotetext{
${ }^{14} \mathrm{Il}$ termine eidolon $5 \mathrm{e}$ usato in Omero esclusivamente in senso deteriore per indicare enti privi di sostanza e di vita. Nell'Iliade XXIII, 104 il termine indica la differenza fra l'ente animato e la sua ombra, un fantasma, una presenza puramente illusoria. Su questo termine si vedano gli studi di VERNANT, 1979, p. 125-126; NAPOLITANO-VALDITARA, 2007, p. 136; GASTALDI, 2007, p. 105 e LASPIA, 2011, p. 112-113, n.6.

${ }^{15}$ Per i principali problemi interpretativi relativi a questa definizione di "immagine", si veda NAPOLITANO VALDITARA, 2007; PALUMBO, 1994, p. 61-3.

${ }^{16}$ Sofista 240a 2-3:

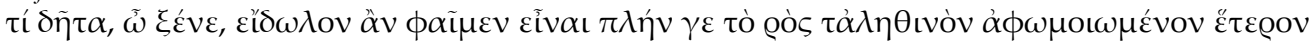

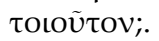

${ }^{17}$ L'espressione toเoṽ tov alla linea 240a7-8 è di difficile traduzione e crea varianti interpretative. Spiega ROSEN "To be "such" means normally to be an instance of a given kind. Since the "kind" in question is the original, one would assume that the original and the "other" are both of the same kind. But this analysis gives rise to a problem of interpretation. Does the "otherness" of the copy derive from its being the same kind as the original? In this case, the kind is a second form standing above both original and copy, and we seem to be on the way toward an infinite regress. Or is the "otherness"due to a distinction between the kind, which is the same in original and copy, and a medium in which the image is displayed? In this second case, the danger of an infinite regress is averted, but at the price of destroying the essential distinction between original and image" (1983, p. 190).

18 Sofista 240b1:

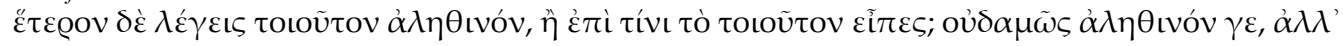
غ̇oukò $\mu \varepsilon \dot{v} v$.

${ }^{19}$ Sull'uso di questo termine, si veda CASERTANO, 2010, p. 172-173. Nel Timeo, Platone utilizza il

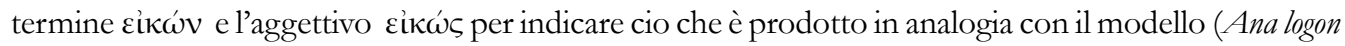


il contrario (enantion) del vero, l'immagine, non essendo vera, non è qualcosa che realmente è, ${ }^{20} \mathrm{ma}$ "di certa forma" è̀ ${ }^{21}$ è non in quanto vera, ma in quanto copia. ${ }^{22}$ Di conseguenza, pur non essendo un ente reale, è realmente ciò che chiamiamo una copia. ${ }^{23}$

Se il passo è estremamente controverso, ${ }^{24}$ il che permette varie interpretazioni, il senso generale del testo è sufficientemente chiaro: la realtà dell'immagine è di essere imitazione dell'originale di cui è copia e del quale conserva lo stesso nome e di essere differente dal modello, infatti non ha la stessa natura di esso. Dal punto di vista ontologico, l'immagine si situa tra il non-essere, perché non è l'ente reale, e l'essere, perché anch'essa, in un certo senso, è. L'immagine non è veramente, perché vero è solo l'ente reale, ma è necessario attribuire anche all'immagine una certa realtà.

È difficile determinare lo statuto ontologico dell'immagine. ${ }^{25}$ Lo stesso Socrate, nella Repubblica, rimane prigioniero di un gioco di parole: "l'immagine del letto è qualcosa come un letto, senza esserlo realmente" (597a). L'immagine è simile al suo modello e differente da esso. Una creazione mimetica acquisisce consistenza, sostiene Teeteto nel Sofista, dall'intreccio fra essere e non-essere. ${ }^{26} \mathrm{I}$ protagonisti del dialogo Sofista attribuiscono realtà all'immagine solo dopo aver riconosciuto l'esistenza del non-essere, la quale è accettata, a patto di non essere intesa come ciò che è assolutamente contrario all'essere, ossia il non-essere assoluto, ma come il "diverso" dell'essere, il suo "altro" (256c-258c). L'immagine di Cratilo è Cratilo, perché non è l'immagine di Simmia, ma è altro di Cratilo, è la sua immagine. ${ }^{27}$

te ekeinon, [29c3), quindi un ente verosimile. Platone osserva: dopo tanti che hanno espresso opinioni diverse sulla nascita e sul divenire di tutto (29c5), non ti meravigliare se non possiamo offrirti discorsi logicamente coerenti ed irreprensibili $(29 \mathrm{c} 6)$. L'importante è che essi non siano meno verosimili $(29 \mathrm{c} 7$ 8: hetton ... eikotas) di quelli degli altri. Il discorso di Timeo nell'omonimo dialogo sottolinea più volte questa verosimiglianza propria del discorso che abbia come oggetto il mondo fisico (eikota mython, $\square$ 29d2), rispetto al suo correlativo matematico che ha come oggetto la struttura intelligibile degli enti. Sul discorso verosimile del Timeo si veda CASERTANO, 1996, p. 270.

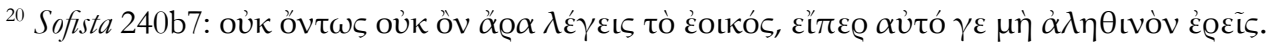
Cordero sopprime oủk óv $\tau \omega \varsigma$ seguendo i manoscritti T e Y (CORDERO, 1993, annesso II, 288). CORNFORD sopprime il secondo oủkس(CORNFORD, s/d, p. 195, n.11).

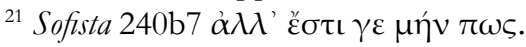

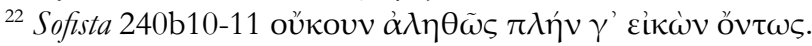

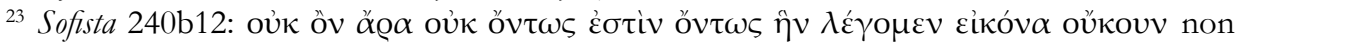
secondo i manoscritti T e Y adottati da CORDERO (1993, p. 238, n169).

${ }^{24}$ Sulle varianti dei manoscritti, si veda CORDERO, 1993, annesso II, p. 288-290. Per l'analisi del passo si veda PEREIRA DA SILVA, 2001, p. 71-78.

${ }^{25}$ Per capire la natura ambigua dell'immagine, è necessario non confondere l'immagine qua somiglianza, con il mezzo attraverso il quale l'immagine è realizzata. Questa distinzione è chiara nel Timeo, in cui il mezzo in cui i phantasmata si concretizzano è la chora. Si veda Timeo 49a-e.

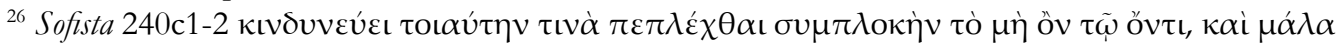
ärotov.

27 " [...] an accurate image contains the same proportions as its original. [...] it is other than that original" (ROSEN, 1986, p. 151). 
La qualità di un'immagine non è determinata dal numero di attributi che la rendono massimamente fedele al modello reale. La differenza fra modello e copia è una differenza di natura e non di grado. Questo è il punto determinante. Pur rappresentando la realtà, l'immagine non è la realtà. Come un ambasciatore diplomatico, l'immagine rappresenta una realtà, perché la presenta e la rende presente ai presenti, pur non essendo la realtà rappresentata.

L'immagine più fedele di Cratilo sempre rimarrà un'immagine e non diventerà mai un secondo Cratilo. "A reflection of a horse is not a horse at all", afferma ripetutamente Patterson nel suo studio dedicato all'immagine in Platone (1985, p. 3). Ė esattamente per il fatto di avere una natura differente dal modello che l'immagine è immagine. Il "principio di non-identità", pertanto, è essenziale al costituirsi dell'immagine. ${ }^{28}$

Ma così come non è corretto spingere la somiglianza tra l'archetipo e la sua copia fino all'identità, è necessario, allo stesso tempo, non eccedere nella differenza. "In un certo senso" (tini tropoi), osserva Platone nella Repubblica (596e), esiste una certa identità tra l'immagine e il modello. C'è dunque una controparte positiva nella relazione tra modello e immagine, come scrive Patterson, "a positive relation between image and model F, and the painter's bed called a bed "in a way" (1985, p. 42). Infatti, si fa riferimento ad entrambe con lo stesso nome. ${ }^{29}$ Non è possibile comprendere la vera natura dell'immagine senza compiere questi due movimenti complementari e opposti: da una lato, un movimento di attrazione che riconosce la somiglianza tra il modello e l'immagine; dall'altro, un movimento di repulsione, che riconosce la differenza fra i due. L'immagine evoca l'originale attraverso una serie di deformazioni essenziali al suo statuto di immagine (LASPIA, 2011, p. 113). Come sottolinea Palumbo, "la valenza positiva delle immagini fedeli consiste proprio nel non occultare questo scarto” (2008, p. 54).

Il principio di non-identità è, pertanto, essenziale al costituirsi dell'immagine, ma ciò che la definisce è l'identità "in un certo senso" con il suo modello. ${ }^{30}$ Scrive Patterson, "The reflection, statue, or painting is a horse only in that it images or imitates, or is a phantasm, eidolon, or ikon of a horse. [...] An image or imitation is not only an image. It is an imitation

\footnotetext{
${ }^{28}$ Questo principio è unanimamente riconosciuto dagli Ellenisti, e messo in evidenza in particolare da BRISSON, 1991; BRUNSCHWIG, 1985, come osserva chiaramente JOUAN, 1996, p. 27.

${ }^{29} \mathrm{Nel}$ Sofista 234b, distinguendo le due specie di arte dell'imitazione (mimetike techne), Platone osserva che la tecnica produttiva produce oggetti che hanno lo stesso nome di altri, ma "non sono" questi altri. Tale rapporto di omonimia è giustificato dall'esistenza del rapporto mimetico. Si veda CASERTANO, 1996, p. 125-126.

${ }^{30}$ Questi due aspetti costitutivi dell'immagine permeano molti dialoghi di Platone e hanno una eco nella definizione di dialettica nel Fedro 265d-e. In questo dialogo, la conoscenza filosofica è caratterizzata della capacità di abbracciare con uno sguardo di congiunto l'identico e il diverso, ossia, di unire in un'unica idea ciò che è molteplice e disgiunto, e distinguere, secondo le giunture naturali, ciò che è unico. Senza contare che, nella Repubblica, è la capacità di distinguere le immagini dai modelli il criterio di elezione di coloro ai quali spetta il diritto di esercitare il potere nella città (476a ss.). Questo punto è stato ben chiarito da FERRARI, 2005, p. 365-391, in particolare p. 365-367.
} 
horse, or a shuttle image" (1985, p. 42). Trattandosi dell'immagine, è necessario che il discorso sempre proceda sul duplice binario del distinguere e del riconoscere.

Nel Politico, Platone confessa come sia impossibile non servirsi di immagini per "mostrare" esaurientemente (bikanos endeiknysthar) ${ }^{31}$ qualcosa di importante" (277d2). Allo stesso tempo, però, il filosofo mette in guardia di fronte al pericolo che il ricorso alle immagini comporta. "C'è il pericolo infatti che ciascuno di noi, sapendo tutto come in sogno, torni poi ad ignorare tutto di nuovo come in un risveglio" (277d). Lo Straniero, il personaggio che conduce il dialogo nel Politico, sottolinea l'ineludibilità di ricorrere all'uso di immagini e la difficoltà che questo espediente comporta. "È necessario individuare quel qualcosa di comune alle immagini che ne costituisce il fondamento e fonda, a sua volta, la conoscenza" (CASERTANO, 1996, p. 250). Comprendere la verità delle cose è per Platone impossibile senza che venga riconosciuta la dipendenza tra l'eidos e la sua immagine, ma in modo tale da non confondere l'uno e l'altra. In questo senso, è significativo l'accenno al sogno e allo stare svegli, ${ }^{32}$ che richiama la nota metafora di Eraclito. ${ }^{33} \mathrm{Nel}$ Sofista $(247 \mathrm{~d})$, Platone ribadisce che se il discorso fosse strutturato solo attraverso immagini e parabole, le nostre conoscenze sarebbero come avvolte nell'indistinzione, e se anche fossimo in possesso di vera conoscenza, essa non avrebbe l'efficacia, né la forza del sapere. ${ }^{34}$

\section{2) L'INFERIORITÀ DELL'IMMAGINE}

Nel passo del Politico anteriormente citato, Platone attribuisce al discorso figurato la capacità di "mostrare" (endeiknysthai, deloo) le cose importanti.

Nuovamente nel Cratilo, la relazione mimetica che corre fra il nome e ciò che il nome indica è prevalentemente dell'ordine del "mostrare". I termini semeion, mimema, deloma sono usati come se fossero sinonimi. A 430b12, il nome è definito "immagine della cosa" (mimema tou pragmatos), mentre a $433 \mathrm{~d} 2$ lo stesso è designato con l'espressione "segno della cosa" (deloma tou pragmatos). A 436e5 i nomi rivelano (semainein), mostrano (deloun) l'essenza dell'ente nominato e a 434a1-2 l'espressione to onomati deloun $\$$ ta ad indicare, secondo l'interpretazione di De Souza, il processo di rappresentare attraverso l'imitazione (2009, p. 175, n14).

Parzialmente d'accordo con De Souza è Lidia Palumbo, la quale difende con forza la necessità di abbandonare la traduzione di mimesis con "imitazione" e adottare, al suo posto, il termine "rappresentazione", da cui si evince, scrive la studiosa, "la potente carica trasformatrice dell'esistente che abita la semantica della mimêsis, così come essa è concepita nei dialoghi di Platone" (2011, p. 166, n32). ${ }^{35}$ Secondo Palumbo, l'idea di Platone è che l'immagine, sia essa un ente sensibile, un'immagine pittorica o un nome, "rappresenta"

\footnotetext{
${ }^{31}$ Anteriormente, Platone aveva usato il verbo deloo $\mathbb{W m o s t r a r e \square}$

${ }^{32}$ Platone utilizza ancora la metafora del sogno in Timeo 52a-b.

${ }^{33}$ DK 22B1, B2, B26, B73, B98.

${ }^{34} \mathrm{Su}$ questo punto si veda CASERTANO, 1996, p. 251, n216.

${ }^{35}$ Sull'opportunità di questa traduzione di veda PALUMBO, 2008.
} 
l'archetipo. "Nel mondo empirico vi sono volti dalle sembianze divine, forme corporee che rappresentano bene la bellezza" (Fedro 251a3). ${ }^{36}$

Secondo Cornford, l'immagine (eikon) deve essere pensata come una fedele "riproduzione" (likeness) del modello. Lo studioso fornisce la seguente giustificazione: "Il termine "immagine" indica una riproduzione o replica (likeness), come la produzione di un secondo letto che riproduce esattamente il primo costruito dal falegname. [...] non esiste alcuna differenza fra la copia (likeness) e l'originale. Entrambe sono identici (exacly alikee) e ciascuno di essi può essere chiamato una immagine (very image) dell'altro" (2003, p. 198). ${ }^{37}$

La giustificazione che Cornford fornisce condanna la sua traduzione per varie ragioni. Anzitutto, ritengo che la scelta del termine "likeness" come traduzione del greco eoikos, eikon Ghon sia felice. Il termine eoikos indica ad un tempo somiglianza e differenza, e si distingue in ciò dal termine homoios. ${ }^{38}$ Forse un'espressione inglese più consona al greco eoikos, eikon é "likened to", oppure "to be a likeness of". ${ }^{39}$ Inoltre, con la sua traduzione, Cornford sembra disconoscere che la relazione fra modello e immagine non è una relazione simmetrica. ${ }^{40}$ Lo studioso afferma che non esiste alcuna differenza fra la copia e l'originale, perciò ciascuno di essi può essere chiamato immagine dell'altro. Questa conclusione prevede che i due enti abbiano ciascuno una propria autonomia ontologica. ${ }^{41}$ Ora, la letteratura specialistica sulla mimesis in Platone è di accordo unanime nel riconoscere che la natura dell'immagine non è solo di essere simile al modello, ma, soprattutto, come chiarisce Palumbo, di essere solo ed esclusivamente questo "essere simile". L'immagine altro non è che questa relazione di somiglianza (PALUMBO 2011, p. 161). ${ }^{42}$

\footnotetext{
${ }^{36}$ Traduzione di Lidia Palumbo. Con l'espressione "bene rappresentano", la studiosa italiana traduce l'espressione eu memimenon $\square$

${ }^{37}$ Traduzione nostra. Un'interpretazione incline ad accettare la lettura di Cornford sembra quella di NEHAMAS (1999, p. 202). Spiegando le ragioni dell'analogia tra poesia e pittura, lo studioso scrive: "la poesia non è solo imitazione nel senso di riproduzione [likeness]" (traduzione nostra).

${ }^{38}$ Cf. Parmenide $132 \mathrm{~d} 5-8$.

${ }^{39}$ Sulla distinzione fra i due termini greci eoikos e homoios e le possibili traduzioni inglesi, si veda PATTERSON, 1985, p. 42.

${ }^{40}$ Sono cosciente che la relazione simmetrica o asimmetrica fra modello e immagine è stata oggetto di lungo dibattito fra gli esperti. Non posso discutere in questa sede le differenti correnti interpretative. Per questo problema rimando a ROSEN, 1983, p. 156, p. 187. Alle pagine 193-200 lo studioso esamina dettagliatamente le interpretazioni di Vlastos, Owen, Cherniss e Lee su questo punto, prima di presentare il suo proprio punto di vista sulla questione.

${ }^{41}$ In verità, qua immagine, l'immagine ha uno status ontologico suo proprio, ossia è realmente una vera immagine, come riconosce lo stesso Platone nel Sofista. Anche se qualcosa non può costituirsi come immagine se non grazie all'esistenza dell'originale, essere un'immagine significa appartenere ad un genere di enti e, in questo senso, possedere una certa indipendenza, in quando distinto da altri generi. Su questo punto si veda ROSEN, 1983, p. 191 e 193.

${ }_{42}$ Sul rapporto di somiglianza al modello che rende l'immagine qualcosa di incompiuto, che rimanda ad altro da sé, cito due studi particolarmente rilevanti: VERNANT, 1987, p. 105-137; DESCLOS, 2000, p. 310-327.
} 
Ciò che distingue l'immagine dal modello è che la prima è necessariamente dipendente dal secondo per la sua esistenza e il suo significato, mentre il primo è indipendente, addirittura assolutamente indipendente, dall'immagine. Detto altrimenti, l'immagine è separata dal modello in un senso "debole", perché, pur non essendo il modello, e in questo senso è separata, dipende necessariamente da esso per la sua esistenza e per la sua identità, e per questa ragione la separazione è debole. Il modello, al contrario, è separato dall'immagine in senso "forte", nel senso che non dipende da nulla per la sua esistenza se non da se stesso. La relazione di "somiglianza" 43 fra immagine e modello è solo unilaterale.

Un passo delle Enneadi di Plotino chiarisce questo tipo di relazione, nel momento in cui propone l'esistenza di due modi di "essere simile". Scrive Plotino:

Ci sono due specie di somiglianza: la somiglianza che esige un elemento identico negli esseri simili; essa esiste tra enti la cui somiglianza è reciproca, perché derivano dallo stesso principio. Il secondo tipo di somiglianza esiste tra due cose di cui una è diventata simile ad un'altra, che è essa stessa primordiale e della quale non si può dire reciprocamente che è simile; questo secondo tipo di somiglianza non esige la presenza di un elemento identico fra loro, ma piuttosto di un elemento differente. ${ }^{44}$

Il primo tipo di relazione si pone fra due enti sensibili. Il secondo tipo di relazione si stabilisce tra due termini di cui il primo è anteriore e indipendente dal secondo, quest'ultimo potendo esistere solo in quanto dipendente dal primo.

Il mimema è solo e semplicemente la relazione di somiglianza con il proprio modello (PALUMBO, 2011, p. 161, n14). Come spiega Goldschmidt: “L'emploi même du terme paradigme semble impliquer nécessairement la ressemblance de la "deuxième espèce" 45 car personne, sans doute, n'aura l'idée de dire que le modèle ressemble à la copie. C'est par le sophisme du semblable (semblable au semblable) que s'opère le glissement de la ressemblance exemplaire ("la deuxième espèce") à la ressemblance reciproque" (1947, p. 44-45).

Se la separazione fra il modello e l'immagine non è reciproca, l'immagine non potrà mai raggiungere l'identità con il modello, ma semmai aspirare ad essa. Riflettendo sulla relazione fra modello e immagine a livello ontologico, Goldschmidt chiarisce lo sforzo di assimilazione dell'immagine sensibile verso il modello intelligibile con le seguenti parole, “Ce sont les sensibles qui s'épuisent en des efforts innombrables et impuissants à reproduire l'éclat de la Forme. La Forme est la plénitude essencielle dans laquelle chaque objet-image

\footnotetext{
${ }^{43}$ Racchiudo l'espressione fra virgolette perché non sono sicura che sia il modo più adatto per indicare la relazione fra il modello e la copia.

${ }^{44}$ Plotino, Enneadi I, 2, 2,4-10

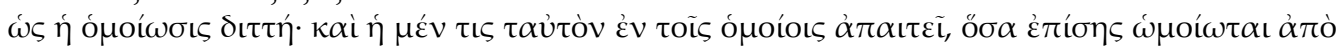

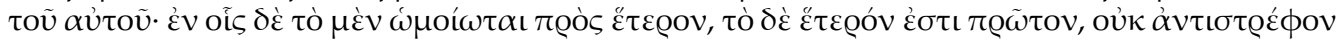

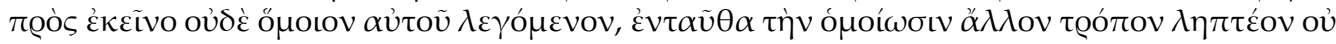

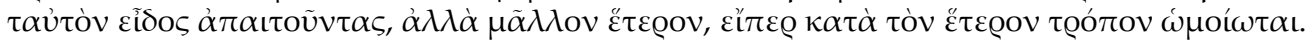

${ }^{45}$ Lo studioso si riferisce alla distinzione operata da Plotino.
} 
ne peut découper que quelques traits, qualités usurpées qu'il ne peut retenir longtemps et sur lesquelles il ne peut jamais faire valoir un droit essenciel” (1949, p. 38).

Se la somiglianza fra modello e immagine fosse reciproca, non si dovrebbe neppure usare il termine "somiglianza" o "copia", bensì il termine "uguaglianza" o "identità", in quanto la relazione con il modello non si limiterebbe a condividere lo stesso nome, secondo il rapporto di omonimia riconosciuto da Platone, ma condizionerebbe l'identità fra gli enti stessi. In tal caso, Platone dovrebbe affermare che l'immagine non è eoikos, somigliante, al modello, ma homoion, identica, ad esso. ${ }^{46}$ Brisson commenta "la chose sensible ressemble à la forme intelligible [...], mais cette ressemblance n'est pas totale sinon chose sensible et forme intelligible seraient identiques" (1992, p. 156).

Nel Fedone l'idea che l'immagine sensibile possa solo tendere ad assimilarsi al modello intelligibile senza mai raggiungere l'identità con esso è resa evidente dall'uso dei verbi: desiderare (bouletai), aspirare (oregetai), avere in vista (prothumeitai):

Questa cosa che io vedo è qualche cosa che vuole essere (bouletai) come un'altra, cioè come uno degli esseri che sono per sé, ma rispetto ad esso è manchevole e non riesce ad essere come quello ed è inferiore [...]. Tutte le cose uguali che percepiamo mediante le sensazioni, tendono (oregetai) ad essere come l'uguale in sé, ma rispetto ad esso sono difettose. [...] ...in quanto tutte queste cose hanno desiderio (prothumeitai) di essere come quello, ma rimangono inferiore ad esso. ${ }^{47}$

\footnotetext{
${ }^{46}$ È esattamente questa la mossa astuta giocata da Parmenide nell'omonimo dialogo, con l'obbiettivo di confutare la proposta del Giovane Socrate. Così facendo, l'idea di Socrate cade nell'aporia del "terzo uomo” . La proposta del Giovane Socrate è la seguente: “... mi sembra preferibile assumere che le Idee stanno nella realtà come modelli e gli altri oggetti assomigliano (eoikemai, eikasthenai) ad esse e sono copie. La partecipazione alle Idee delle altre cose non consiste in altro che nell'essere fatte a immagine (homoiomata) di quelle". Parmenide risponde riprendendo le parole di Socrate, ma non senza un leggero glissement che produce un effetto di simmetria. Come suggerisce giustamente PATTERSON (1985, p. 58), Parmenide sostituisce surrettiziamente ai termini di Socrate che esprimono la somiglianza non simmetrica delle copie ai loro modelli un termine che indica una similitudine simmetrica, ossia una forma di uguaglianza: "Se dunque qualcosa assomiglia (eoiken) all'Idea è possibile che questa Idea non sia simile (bomoion) alla sua immagine, [...]? O c'è un qualche espediente per cui il simile (to homoion)

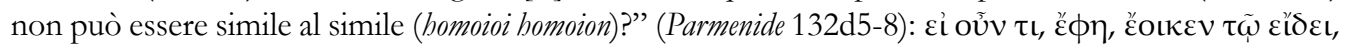

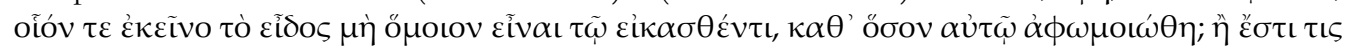

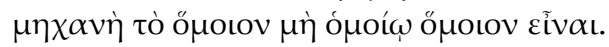

${ }^{47}$ Fedone 74d-75d口

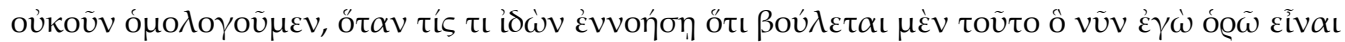

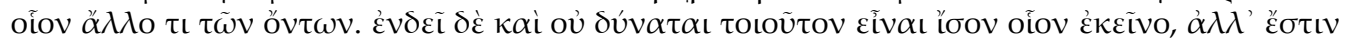

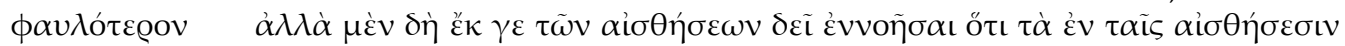

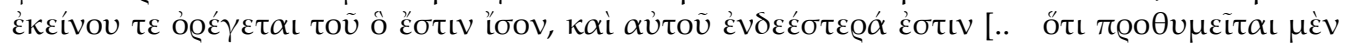

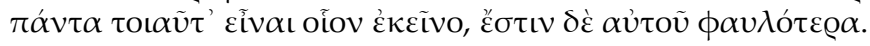


Nel cosmo platonico lo sforzo di assimiliazione al modello che anima gli enti del mondo sensibile è evidente in tutti i livelli, dal bastoncino di legno dell'esempio del Fedone che "aspira" all'uguaglianza ideale (74c), fino all'uomo giusto che ha come suo parametro il paradigma divino: "Dio in nessuna circostanza, in nessun modo, è ingiusto, ma, anzi, è supremamente giusto, e non c'è niente di più simile a lui di quello di noi che, a sua volta, sia diventato il più giusto possibile" (Teeteto 176c)..$^{48}$

La somiglianza intesa come sforzo di assimilazione si armonizza con l'origine del termine mimesis. Questo sostantivo deriva dal verbo mimeomai, il quale, a sua volta trova origine dal nome del mimo-attore, mimos. Scrive Else, "the original sphere of mimêsis - or rather mîmos and mimeîsthai - was the imitation of animate beings, animal and human, by the body and the voice (...), rather than by artefacts such as statues or sculptures. In other words, these terms originally denoted a dramatic or quasy-dramatic representation, and their extension to non-dramatic form like painting and sculpture must have been a secondary development." (1958, p. 78). Lo stesso giudizio è condiviso da R. Dupont-Roc e J. Lallot che, nell'introduzione alla traduzione e commento della Poetica di Aristotele, scrivono: "La famille de mimesis s'enracine dans une forme de représentation au sens théâtrale du mot" (2011, p. 18), e da Havelock: "... mimesis ... a notion derived from the specific context of mimicry and dramatic representation" (1963, p. 27$).{ }^{49}$

Il significato originario del termine, pertanto, è attivo,ossia, rendersi simile al modello, rappresentare mediante atteggiamenti del corpo, gesti e espressioni del viso, come fanno i mimi. Nehamas (1999, p. 206) conferma che originariamente il termine mimesis è usato solo per caratterizzare la poesia teatrale, drammatica e la danza e significa principalmente agire come [acting like] un'altra persona, agire come se si fosse un'altra persona.

Un mirabile esempio di quanto è stato evidenziato dagli studiosi è il canto del coro delle Deliadi nell'Inno ad Apollo di Pindaro, il quale è raffigurato come una mimesis dei linguaggi inintelligibili di tutti gli uomini. ${ }^{50} \mathrm{Si}$ tratta di un riprodurre artisticamente, di fronte al pubblico degli Ioni riuniti nell'isola in occasione della grande festa, linguaggi non propri: un far rivivere in modo da coinvolgere anche emozionalmente i convenuti. ${ }^{51}$

Il senso passivo, essere una imitazione, ossia essere risultato di un lavoro di imitazione e copia di un modello, è un senso derivato e tardo.

È utile osservare, infine, che il verbo in questione, mimeomai, è impiegato da Platone sempre in vox media. La vox media, diversamente dalle voci attiva e passiva, riunisce in sé le due condizioni dell'agire e del patire. Mi pare ragionevole pensare che il "rendersi simile"

\footnotetext{
48 Teeteto 176c: $\square$

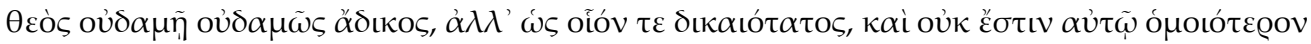

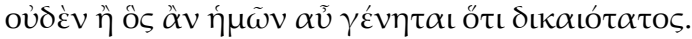

${ }^{49}$ L'autore ne parla diffusamente nelle pagine 20-35.

${ }^{50}$ Pindaro, Inno ad Apollo, v. 163 ss.

${ }^{51}$ Gli esempi a questo rispetto potrebbero moltiplicarsi, ricordo solo il significativo passo tratto dalle Coefore di Eschilo, v. 564 ss.
} 
(mimeisthai), si dica alla vox media perché il soggetto compie l'azione di tendere al modello ed è, allo stesso tempo, modificato dal modello che rappresenta e che gli dá senso ed esistenza.

L'aspetto originario del termine appare nel Sofista. Dopo aver presentato la tecnica mimetica (eidolopoïhe) nella sezione 235b-d, Platone la riprende verso la fine del dialogo. In 265b, la sua determinazione viene compiuta attraverso una nuova divisione della tecnica produttiva in produzione umana attraverso l'uso di strumenti, o usando se stessi e il proprio corpo e la propria voce. Questa seconda parte, sottolinea espressamente Platone, è "propriamente chiamata" (malista kekletai) imitazione (mimesis, mimetikon). Come si vede, il termine mimesis è propriamente l'accezione che indica l'atto di produrre immagini con il proprio corpo e con la propria voce. ${ }^{52}$

Gli scrittori latini alludono allo stesso significato, visto che in alcuni testi filosofici latini il verbo mimeomai è reso dal latino simulare. Come osserva Pradeau, è evidente che $\mathrm{i}$ latini prediligono simulare ad imitare. Cicerone, per esempio, scrive: "Ne quid fictum sit neve simulatum", ossia, l'uomo dabbene deve evitare tutto ciò che sarebbe finto o simulato; e poco più avanti nello stesso testo, ciò che è simulato si oppone a ciò che è verum et voluntarium. ${ }^{53}$

Queste sono alcune delle ragioni che mi hanno spinto a credere che funzione principale dell'“immagine" non sia quella di imitare.

\section{3) LA MIMESIS, MEDIUM DELLE REALTÀ ASSENTI}

L'immagine in Platone e nella letteratura greca arcaica è presente quando si tratta di evocare e di vedere qualcosa di per sé assente, e che senza la mimesis non potrebbe essere immaginato.

Se i Greci sentono la presenza della divinità al cospetto della statua di Atena, è giustamente per il fatto che la dea è assente, nella misura in cui supera infinitamente ciascuna delle forme attraverso le quali l'artista ha cercato di concretizzarne la presenza. ${ }^{54}$ La realtà della statua è di essere un ente privo di identità che acquista senso nell'essere "immagine di". "Ecco la situazione base del fenomeno mimetico: l'ente ospitante è una mimesis o un mimema dell'ente superiore ospitato" (PALUMBO, 2011, p. 171). Il legame tra l'archetipo e la sua copia è un legame fatto di rimandi e di riflessi, in cui è importante sovrapporre, ma anche distinguere.

\footnotetext{
${ }^{52}$ Per la presenza del senso emulativo di mimesis nella Repubblica si veda NEHAMAS, 1999, p. 224.

${ }^{53}$ CICERONE, L'Amicizia XVIII 65; si veda anche VIII 26; XXV 95. Cf. PALUMBO, 2011, p. 165, n29. PRADEAU (2009, p. 66) rileva che il latino simulacrum traduce il greco eidolon $\square$

${ }^{54} \mathrm{Nel} \mathrm{Fedro,} \mathrm{Platone} \mathrm{afferma} \mathrm{che} \mathrm{diamo} \mathrm{il} \mathrm{nome} \mathrm{di} \mathrm{immortale} \mathrm{alla} \mathrm{divinità,} \mathrm{figurandocela,} \mathrm{pur} \mathrm{senza} \mathrm{vederla,}$ come un vivente dotato di un'anima e di un corpo che non si staccano mai: "Il termine "immortale" non può essere spiegato sulla base di un solo discorso razionale; ma senza conoscerlo ed intenderlo in modo adeguato, noi ci figuriamo un dio, un essere vivente e immortale, che ha un'anima e un corpo eternamente connaturati” (246d). Su questo punto si veda JOUAN, 1996, p. 106.
} 
Nella tragedia Alcesti, Euripide descrive l'emozione di Admeto diante la statua che evoca la presenza della donna amata: ${ }^{55}$

Raffigurato (eikasthen) da mani di abili artisti il tuo corpo sarà disteso sul mio letto; accanto a lui mi coricherò, e tendendo le mani, invocando il tuo nome, è la mia sposa che crederò di abbracciare, benché assente. Fredda voluttà, certo, e che tuttavia allevierà il peso del mio cuore (v. 348-352). ${ }^{56}$

Nella misura in cui si gioca sull'ambiguità essere-apparire, la seduzione esercitata dall'immagine coinvolge ad un tempo la parte razionale e quella epithymetica, desiderativa, dell'anima ${ }^{57}$ Questa è la ragione per cui l'immagine emana un flusso emotivo. Non esiste immagine, neppure una semplice copia, che non susciti un'emozione e non esiste raffigurazione, neppure la più astratta, dalla quale non scaturisca un'idea.

Presenze impalpabili, quella di Alcesti e della dea Atena, che ad ogni tentativo di approssimazione fisica svaniscono nell'assenza, come le ombre che Ulisse incontra durante la sua discesa all'Ade. ${ }^{58}$ È la madre, Anticlea, che nell'Ade riconosce il figlio, manifestandosi nella voce e nei gesti. Ma quando Ulisse si lancia verso di lei nel tentativo di stringerla tra le braccia, si scontra con un doloroso vuoto:

Venne mia madre e bevve il sangue nero; subito mi riconobbe e gemendo mi disse alate parole. [...] Esitando nell'animo, volevo prendere tra le braccia l'anima di mia madre defunta. Tre volte mi lanciai e l'animo mio mi spingeva a prenderla: tre volte simile a ombra o a sogno dalle braccia volò via; e a me ancor più nel cuore nasceva acuta pena (Odissea, XI, v. 206-208). ${ }^{59}$

\footnotetext{
${ }^{55}$ Sulle emozioni provocate dalle creazioni plastiche e artistiche, in generale, si veda GOMBRICH, 1961.

${ }^{56}$ Traduzione personale.

${ }^{57}$ Si veda VILLELA-PETIT, 1991, p. 63. Su questo aspetto è interessante anche lo scritto di VERNANT, 1979, p. 133-160. L'azione della poesia mimetica sulla parte irrazionale dell'anima è argomento del libro X della Repubblica, in particolare alle pagine 595b-606d. In queste pagine, Platone distingue espressamente una facoltà superiore dell'anima, alla quale è proprio il calcolo e la misura, da una parte inferiore, chiamata to anoeton, la quale si lascia affascinare dalle apparenze (602a-605b). Nel Sofista 234b, il filosofo utilizza lo stesso aggettivo anoetous[per indicare chi si lascia ingannare dall'arte

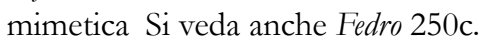

${ }^{58}$ Si veda Omero, Odissea, XI, v. 153-222.

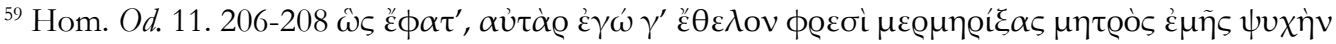

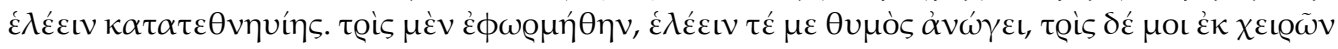

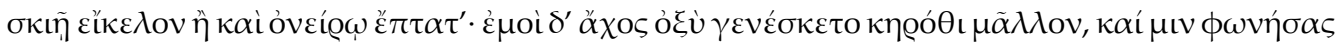

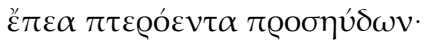


La mimesis è l'espediente que evoca enti privi di forma concreta e li trasforma in immagini visibili agli occhi non sensibili. È interessante far risaltare che nel canto XI dell'Odissea le anime di Tiresia, della madre di Ulisse e dei suoi compagni morti, sono dette eidola. ${ }^{60}$

Achille fa la stessa esperienza di Ulisse con l'immagine del valoroso Patroclo nell'Iliade:

Ed ecco Comparirgli del misero Patroclo In vision lo spettro, a lui del tutto Ne' begli occhi simile e nella voce, Nella statura, nelle vesti [...] Coll'aperte braccia Amoroso avventossi, e nulla strinse, Chè stridendo calò l'ombra sotterra, E svanì come fumo. In piè rizzossi Sbalordito il Pelide, e palma a palma Battendo, in suono di lamento disse: Oh ciel! dell'Orco gli abitanti han dunque Spirito ed ombra, ma non corpo alcuno? Del misero Patroclo in questa notte Sovra il capo mi stette il sospiro (Iliade, XXIII, v. 65-108). ${ }^{61}$

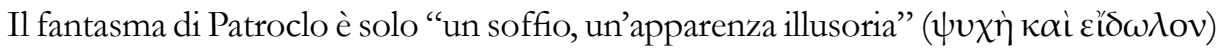

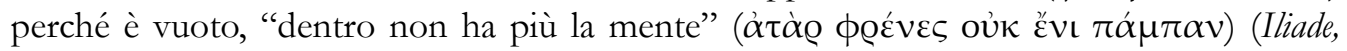
XXIII, v. 104).

I versi omerici, vibranti e commoventi, rimandano il lettore al proemio del Fedone, in cui il discepolo di Socrate confessa che parlare del Maestro e sentire parlare di lui sono "la più dolce di tutte le cose" (58d5-6). ${ }^{62}$

L'approssimazione tra l'arte visiva, la parola e l'immagine ${ }^{63}$ non è nuova nella letteratura greca. La relazione tra logos e eidolon, mimema o eikon si trova, per esempio, negli oratori. Alcidamante, nell'orazione Sugli autori di discorsi scritti, ovvero sui sofisti, ai versi 27 -

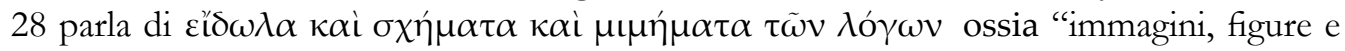
rappresentazioni di discorsi”. ${ }^{64}$

\footnotetext{
${ }^{60}$ Cf. Omero, Odissea, XI, v. 174; v. 476 ss.

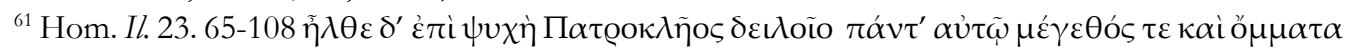

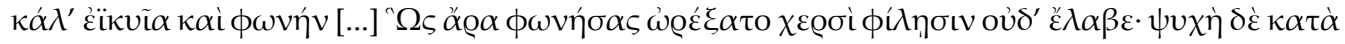

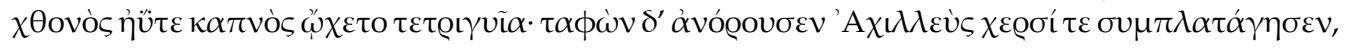

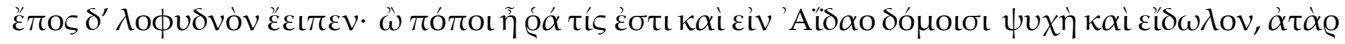

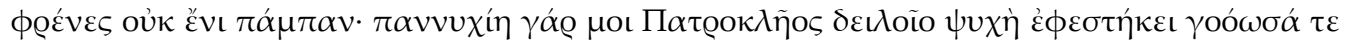

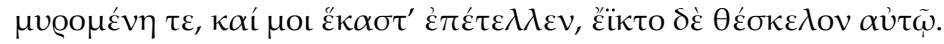

${ }^{62}$ Su questo passo del Fedone, si veda PALUMBO, 2003, p. 291-300.

${ }^{63}$ Platone scivola con disinvoltura dalla pittura al discorso in Sofista 239e5-240a6. Commentando queste pagine, ROSEN osserva "The sophist forces us to shift from visual images to the many senses of image that follow from the discurse" (1986, p. 151). In che senso il discorso è una immagine è chiarito da ROSEN in questi termini "To say what is, or what is not, is to copy what is, accurately or inaccurately, not in the literal sense of "painting" it, but in the sense of representing by the words and syntax of natural language (the logical form) the intellible or structural elements of what is" (1986, p. 155).

${ }^{64}$ Sulla relazione tra discorso e immagine, si veda LASPIA, 2011, p. 112, n4. TRABATTONI (2005, p. 22-29) e VELARDI (2006, p. 302-303) trovano echi simili in Isocrate.
} 
Nel Fedro, Socrate scrive che la scrittura è simile alla pittura (zographia). Tanto un dipinto quanto un discorso sono manifestazioni di eidola (275d-276a). ${ }^{65} \mathrm{Nel}$ Sofista, il filosofo osserva che accanto alle imitazioni prodotte dal pittore (mimemata ... ton onton, [234b6-7), anche a proposito dei discorsi esiste una tecnica (peri tous logous ... einai tina allen technen[234c2-3) che, mostrando immagini verbali (eidola legomena peri panton[234c6) seduce e fa sembrare che si sia detto cose reali (alethe dokein legesthai[234c6-7).

Nel caso di poesia e pittura, ${ }^{66}$ Platone riprende l'analogia, attribuita a Simonide, tra i due tipi di manifestazione artistica e rinforza così una tradizione che sarà rinnovata nella Poetica di Aristotele e nella ut pictura poesis, secondo l'espressione latina, della Ars Poetica di Orazio. ${ }^{67}$

La differenza essenziale fra l'archetipo e la copia è che l'originale ha vita, è animato, l'immagine no. La creatura dipinta, osserva Socrate nel Fedro, ti sta davanti come se fosse viva (hos zonta) ma se le rivolgi la parola mantiene un solenne silenzio (semnon pany sigai). Lo stesso dicasi per i discorsi (275d). Alcidamante lamenta il fatto che il discorso scritto non persuade e Platone rimprovera al discorso "immobile" di non insegnare (legein) ingannando chi voglia imparare (manthanein), perché dà l'impressione di sapere qualcosa (bos to phronountas) ma poi abbandona il suo interlocutore, come un fantasma (eidolon) evanescente sfuma, si dirada (Fedro 275d-276a). ${ }^{68}$

L'immagine non ha vita, né identità. Essa è un ente privo di sé che ospita nel suo intimo un'altra realtà, della quale l'immagine emana la presenza. In questo modo si comportano un attore sulla scena di teatro, un poeta ispirato dalla Musa divina, una parola animata dal significato, o gli enti fisici da cui emana la realtà intelligibile. L'immagine, il poeta, l'attore, la parola, la realtà sensibile mimountai, ossia evocano kata mimesin, Zina realtà altra, superiore ad essi, che emana da essi, dalla quale dipendono e che dà loro vita, senso e nome.

Platone nelle Leggi scrive che il poeta è spoglio di sé, ossia manca di identità, al fine di indossare un'alterità che deve evocare senza mai potersi identificare con essa: "Un poeta, quando è seduto sul tripode della Musa, perde il controllo di sé e, come una sorgente, lascia liberamente scorrere ciò che gli passa per la mente. La sua è un'arte basata sulla mimesis" (719c3-d1).

E nel Timeo (49e), il filosofo conferma al livello ontologico che nessuno degli enti sensibili è un se stesso, ma ognuno di essi evoca (phantaz̨etai) l'intelligibile.

In Platone l'immagine evoca, rinvia, segnala, emana una realtà assente: l'immagine "rimanda" (semainei, Cratilo 434a1) al modello, perché direziona il pensiero ad esso, evocandone la presenza. L'immagine è il medium che permette l'ascesa dell'anima ad una dimensione "altra".

\footnotetext{
${ }^{65}$ Si veda AVEZZÙ, 1982, p. 71-78. SCHUHL (1952, p. XI-XII) osserva giustamente che l'estrema sensibilità di Platone in relazione alle forme di arte, l'uso preciso del linguaggio dei pittori, del vocabolario tecnico degli ateliers indicherebbe non solo il fascino che l'arte ha esercitato sul filosofo durante la sua giovinezza, ma anche la probabilità che lo stesso Platone si sia cimentato in queste forme di creazione artistica.

${ }^{66}$ L'analogia della pittura con la poesia è argomento del libro X della Repubblica, a partire da 603c.

${ }^{67} \mathrm{Si}$ veda VILLELA-PETIT, 1991, p. 60.

${ }^{68}$ Cf. LASPIA, 2011, p. 103.
} 
La lingua greca ha un sostantivo molto significativo in questo senso. Il termine sema\acchiude in un'unica parola un'idea e un'emozione, una ricchezza sconosciuta al suo corrispondente neolatino. Il termine greco significa ad un tempo "segno" e "pietra tombale", 69 in quanto il sepolcro è immagine dell'invisibile, dell'al di là che solo può essere evocato, ma non rappresentato e, che senza la mimesis non potrebbe essere immaginato.

\section{ConClusione}

Il termine "immagine" è un termine chiave per la lettura e la comprensione della filosofia platonica.

L'immagine rimanda alla particolare relazione che esiste tra la realtà e la sua rappresentazione poetica, ontologica e linguistica.

La relazione che esiste tra la realtà, ideale o empirica, e la sua immagine non può essere ridotta a ciò che chiamiamo "imitazione", sebbene questo significato non sia escluso da essa. Mimesis è la rappresentazione di un mondo e di una possibilità di significato. Tale rappresentazione è mimetica in quanto riesce a coinvolgere emotivamente lo spettatore, inducendolo a confondere l'immagine con la realtà stessa, al punto da scontrarsi con un doloroso vuoto quando egli cerchi di catturare l'essenza dell'immagine.

È caratteristico dell'immagine la dimensione del mancare: l'immagine non risponde alla propria essenza. Essa è ciò che è, ossia immagine, in quanto è in qualche modo in difetto. L'immagine è qualcosa come un ente senza esserlo relamente. La sua unica forza è la sua efficacia comunicativa, il suo potere di seduzione, in quanto essa agisce ad un tempo a livello razionale ed emotivo.

Nel presente studio, ho cercato di evidenziare questa duplice dimensione dell'immagine: da un lato, la sua dipendenza da un modello reale che dà vita, identità e significato all'immagine, sebbene l'immagine non possa mai raggiungere l'identità con la realtà da cui dipende; dall'altro la carica emotiva racchiusa in ogni immagine, la quale mostra agli occhi sensibili o non sensibili una realtà assente che attraverso l'immagine si rende a tal punto presente, che lo spettatore vive "come in un sogno", ossia fa coincidere l'interezza dell'esperienza con questo "vedere".

Platone era consapevole dello straordinario potere evocativo delle immagini e di come tale potere sia utile ad approssimare la vita alla poesia e al teatro, luoghi della mescolanza di emozione e di pensieri.

\section{REFERÊNCIAS}

ARISTOTE. La Poétique. texte, traduction, notes par Roselyne Dupont-Roc et Jean Lallot. Paris: Éditions du Seuil, 2011.

\footnotetext{
${ }^{69}$ Per uno studio del segno religioso si veda VERNANT, 1970.
} 
AVEZZÙ, G. Alcidamante: Oraz̧ioni e Frammenti. <Bollettino dell'Istituto di Filologia greca> Suppl. 6, Roma, 1982.

BRISSON, L. Platon, Timée, Critias. Paris: Flammarion, 1992.

BRISSON, L. Participation et prédication chez Platon, < Revue Philosophique de la France et de l'Étranger> n. 4/1991, p. 557-569.

BRUNSCHWIG, J. Le problème de la "self-participation" chez Platon. In: L'art des confins, Mélanges offerts à M. de Gandillac. Paris: Vrin, 1985. p. 121-135.

CASERTANO, G. La struttura del dialogo (o di quando la filosofia si fa teatro). In:

(Org.). Il Protagora di Platone: Struttura e problematiche. Napoli: Loffredo, 2004. p. 728-766.

CASERTANO, G. Paradigmas de Verdade em Platão. São Paulo: Loyola, 2010.

CASERTANO, G. Caratteristiche e funzioni del Logos. Sulla forma e la struttura del Teeteto. In: MIGLIORI, M. (Org.). Il dibattito etico e politico in Grecia tra il V e il IV secolo. Napoli: La Città del Sole, 2000. p. 337-383.

CASERTANO, G. Uma introdução à Republica de Platão. São Paulo: Paulus, 2011.

CASERTANO, G. Il nome della cosa. Napoli: Loffredo, 1996.

CORDERO, N-L. Introduction in: Platon, Le Sophiste. trad. N. L. Cordero, Paris: GF-Flamarion, 1993.

CORNFORD, F. M. Plato's Theory of Knowledge: the Theaetetus and the Sophist. New York: Dover Publications, 2003.

CORNFORD, F. M. La teoria platonica del conocimiento, traduzione N. L. CORDERO e M. D. C. Ligatto. Buenos Aires: Paidós, s/d.

DE SOUZA, E. C. Discurso e Ontologia em Platão. Um estudo sobre o Sofista. Ijuí: Editora Unijuí, 2009.

DESCLOS, M. L. Idoles, icones et phantasmes dans les dialogues de Platon, < Revue de Métaphysique et de Morale> 3, p. 310-327, 2000.

DIXAUT, M. Métamorphoses de la dialectique dans les dialogues de Platon. Paris: Vrin, 2001.

FERRARI, F.Teoria delle idee e ontologia. In: VEGETTI, M. (Org.). Platone, Repubblica, vol. IV (libro V). Napoli: Bibliopolis, 2005.

FOLSCHEID, D.; WUNENBURGER, J.J. Metodologia filosófica. São Paulo: Martins Fontes, 1997.

GASTALDI, S. La mimesis e l'anima. In Platone, la Repubblica, traduzione e commento a cura di M. VEGETTTI. Vol. VII (libro X). Napoli: Bibliopolis, 2007.

GEBAUER, G.; WULF, C. Mimesis Culture-Art-Society. Los Angeles: University of California Press, 1995. 
GERSON, L. P. God and Greek Philosophy. Studies in the early history of natural theology. LondonNew York: Routledge, 1990.

GOLDSCHMIDT, V. Le paradigme dans la dialectique platonicienne. Paris: Vrin, 1947.

GOLDSCHMIDT, V. La Religios de Platon. Paris: Vrin, 1949.

GOMBRICH, E. H. Art e Illusion. Princeton: Princeton University Press, 1961.

GOMBRICH, E. H. História da Arte. São Paulo: Círculo do Livro, 1972.

GORDON, J. Turning toward Philosophy. Literary Device and Dramatic Structure in Plato's Dialogues. Pennsylvania: The Pennsylvania University Press, 1999.

HALLIWELL, S. The Aestetics of Mimesis. Ancient Texts and modern problems. Oxford and Princeton: Princeton University Press, 2002.

HAMM, C. V. Platão como artista. Archai, n. 12, p. 61-67, jan.-jun. 2014. DOI: <http:// dx.doi.org/10.14195/1984-249X_12_6>.

JOUAN, S. Le problem de la participation chez Platon. Memoire de D.E.A. Université Ch. De Gaulle - Lille 3, 1996.

LASPIA, P. Il discorso dipinto. Scrittura, voce e livelli di significazione a partire dal Fedro di Platone. In: CASERTANO, G. (Org.). Il Fedro di Platone: Struttura e problematiche. Napoli: Loffredo 2011. p. 95-111.

MIGLIORI, M. Il Sofista di Platone. Valore e limiti dell'ontologia. Brescia: Morcelliana, 2006.

NAPOLITANO VALDITARA, L. M. Platone e le "ragioni dell'immagine". Percorsi filosofici e deviazioni fra metafore e miti. Milano: Adelphi, 2007.

NEHAMAS, A. Virtues of Autenticity: Essays of Plato and Socrates. New Jersey: Princeton University Press, 1999.

PALUMBO, L. Mimesis ed enthousiasmos in Platone. Appunti sul Fedro. In Casertano G. (Org.). Il Fedro di Platone: Struttura e Problematiche. Napoli: Loffredo, 2011.

PALUMBO, L. Il non essere e l'apparenza. Sul Sofista di Platone. Napoli: Loffredo, 1994.

PALUMBO, L. Mimesis. Rappresentazione, teatro e mondo nei dialoghi di Platone e nella Poetica di Aristotele. Napoli: Loffredo, 2008.

PALUMBO, L. Le emozioni e il pensiero nel Fedone di Platone. In: VENDITTI (Org.). La filosofia e le emozioni. Atti Del XXXIV Congresso Nazionale della Società Filosofica Italiana. Firenze: Le Monnier, 2003. p. 291-300.

PATTERSON, R. A. Image and Reality in Plato's Metaphysics. Indianapolis: Hackett Publishing Company, 1985.

PEREIRA DA SILVA J. A definição de imagem no Sofista de Platão. Cadernos de Atas da ANPOF, p. 71-78, 1/2001. 
PERINE, M. Il Fedro: un invito alla filosofia. In: CASERTANO, G. (Org.). Il Fedro di Platone: Struttura e Problematiche. Napoli: Loffredo, 2011.

PLOTINO. Ennéades. Trad. E. Bréhier, Coll. Des Universités de France (G. Budé). Paris: Les Belles Lettres, 1924.

POLLIT, J. J. The Ancient View of Greek Art: Criticism, History and Terminology. New Haven \& London: Yale University Press, 1974.

PRADEAU, J.-F. Platon, l'imitation de la philosophie. Paris: Aubier, 2009.

ROSEN, S. Plato's Sophist: the drama of original and image. New Haven and London: Yale University Press, 1983.

SCHUHL, P.-M. Le merveilleux. La pensée et l'action. Paris: Flammarion, 1952.

SPINA, L. L'enárgheia prima del cinema: parole per vedere. In: <Dioniso> 4, p. 298-209, 2005.

TRABATTONI, F. Oralità e scrittura in Platone e nella cultura greca classica. Roma: Carocci, 2005. VELARDI. Platone, Fedro. Milano: BUR, 2006.

VERNANT, J. P. Image et apparence dans la théorie platonicienne de la "mimesis". In: . (Org.). Religions, histoires, raisons. Paris, 1979. p. 133-160.

VERNANT, J. P. Image et apparence dans la théorie platonicienne de la mimêsis (1975) ripubblicato in Naissance d'images, in Religions, histories, raison. Paris: Maspero, 1987. p. 105-137.

VERNANT, J. P. Mito e pensiero presso i Greci: studi di psicologia storica (Mythe et pensée chez les grecs: études de psychologie historique, 1965 e n. ed. 1988). Torino: Einaudi, 1970.

VLASTOS, G. Platonic Studies. Princeton: Princeton University Press, 1973.

VILLELA-PETIT, M. La question de l'immage artistique dans le Sophiste. In: AUBENQUE, P. (Dir.). Etudes sur le Sophiste de Platon. Napoli: Bibliopolis, 1991. p. 53-91.

Recebido em: 18 de novembro de 2014 Aprovado em: 9 de dezembro de 2015 